\title{
Indicated prevention interventions for anxiety in children and adolescents: a review and meta-analysis of school-based programs
}

\author{
Siobhan Hugh-Jones ${ }^{1}\left[\right.$ [D Sophie Beckett ${ }^{2} \cdot$ Ella Tumelty $^{2} \cdot$ Pavan Mallikarjun $^{2}$
}

Received: 9 June 2019 / Accepted: 21 May 2020 / Published online: 13 June 2020

(c) The Author(s) 2020

\begin{abstract}
Anxiety disorders are among the most common youth mental health disorders. Early intervention can reduce elevated anxiety symptoms. School-based interventions exist but it is unclear how effective targeted approaches are for reducing symptoms of anxiety. This review and meta-analysis aimed to determine the effectiveness of school-based indicated interventions for symptomatic children and adolescents. The study was registered with PROSPERO [CRD42018087628]. We searched MEDLINE, EMBASE, PsycINFO, and the Cochrane Library for randomised-controlled trials comparing indicated programs for child and adolescent (5-18 years) anxiety to active or inactive control groups. Data were extracted from papers up to December 2019. The primary outcome was efficacy (mean change in anxiety symptom scores). Sub-group and sensitivity analyses explored intervention intensity and control type. We identified 20 studies with 2076 participants. Eighteen studies were suitable for meta-analysis. A small positive effect was found for indicated programs compared to controls on selfreported anxiety symptoms at post-test $(g=-0.28, \mathrm{CI}=-0.50,-0.05, k=18)$. This benefit was maintained at $6(g=-0.35$, $\mathrm{CI}=-0.58,-0.13, k=9)$ and 12 months $(g=-0.24, \mathrm{CI}=-0.48,0.00, k=4)$. Based on two studies, $>12$ month effects were very small $(g=-0.01, \mathrm{CI}=-0.38,0.36)$. No differences were found based on intervention intensity or control type. Risk of bias and variability between studies was high $\left(I^{2}=78 \%\right)$. Findings show that school-based indicated programs for child and adolescent anxiety can produce small beneficial effects, enduring for up to 12 months. Future studies should include long-term diagnostic assessments.
\end{abstract}

Keywords Child $\cdot$ Adolescent $\cdot$ School-based interventions $\cdot$ Anxiety $\cdot$ Early intervention $\cdot$ Meta-analysis

\section{Introduction}

Electronic supplementary material The online version of this article (https://doi.org/10.1007/s00787-020-01564-x) contains supplementary material, which is available to authorized users.

Siobhan Hugh-Jones

s.hugh-jones@leeds.ac.uk

Sophie Beckett

SXB1131@student.bham.ac.uk

Ella Tumelty

EHT563@student.bham.ac.uk

Pavan Mallikarjun

p.mallikarjun@bham.ac.uk

1 School of Psychology, University of Leeds, Leeds LS2 9JT, UK

2 The Barberry National Centre for Mental Health, University of Birmingham, Birmingham B15 2FG, UK
Globally, approximately 117 million children and young people are affected by anxiety disorders [1]. Fewer than $20 \%$ of young people with anxiety disorder access support [2], and of those that do, a significant minority end treatment prematurely or do not benefit $[3,4]$. Cost-effectiveness analyses indicate that treatment alone is insufficient to eliminate the disease burden of these disorders and that investment in prevention and early intervention are needed [5]. Evidence-based prevention, early intervention and treatment approaches exist for anxiety [6] with broadly similar content, only differing substantively in terms of the time point at which they are delivered [7]. Elevated anxiety symptoms affect quality of life and are a risk factor for anxiety disorders. The reduction of elevated symptoms is therefore important to improve functioning and well-being, and to potentially prevent new cases of disorder. Schools are 
considered good sites for early intervention programs given their reach and circumventing of common barriers to support [8]. Programs can be universal (delivered to all pupils) or targeted, being either selective (delivered to those at higher risk, based, for example, on family history), or indicated (delivered to those with detectable but sub-clinical symptoms) [9]. Most studies evaluate interventions in terms of their impact on anxiety symptoms rather than diagnostic outcomes, possibly due to the resource requirements for diagnostic assessments. It is unclear which delivery approach is most effective for reducing symptoms of anxiety and which should be implemented in schools.

Meta-analyses have reviewed the effects of different delivery formats. Interpretations of the value of reported effect sizes (ES) from this body of work need to consider a number of factors [10]. For example, the importance of an effect can depend on intervention costs, ease of delivery, scale-up penalties, school attendance and academic/social benefits, changes in health care utilisation, numbers of new cases averted and spill over effects to peers [10]. Small ES from relatively inexpensive, scalable interventions (e.g., in schools where the infrastructure exists), that reach large sections of the population at a time of vulnerability to mental health disorder could have substantial practical importance, considering the high prevalence of anxiety, its psychosocial and economic impact, and that so few young people access support via other means [2].

Most school-based anxiety interventions to date have been universal and highly heterogeneous. Meta-analyses of these report small-to-moderate post-test ES in the range of $0.2-0.5$ on anxiety symptoms in children and adolescents [11-20]. Although 12 month effects from universal programs have been reported for depressive symptoms [10, 21], similar outcomes from universal programs for anxiety symptoms are rare, with only a few studies reporting ES spanning small, marginal, or no effects at 12 and/or 24 months [12, 14-17, 19, 20]. Targeted approaches for depression, delivered in school to at-risk young people, appear more effective than universal approaches, suggesting that intervention effectiveness increases with symptom severity [21, 22] However, findings from studies of targeted approaches to reduce anxiety symptoms are mixed. Lawrence, Rooke, and Creswell's (2017) meta-analysis of 16 RCTs, which merged indicated and selective trials, reported a significant smallto-moderate effects ( $\mathrm{ES}=-0.43)$ on post-test anxiety symptoms compared to waitlist controls $(k=10)$ but only a very small non-significant effect compared to attention controls $(\mathrm{ES}=-0.09, k=5)$ [15]. Effects increased up to 6 months but fell at 12 and 24 months.

Two reviews have compared universal and targeted program effectiveness. Merging studies of indicated and selective interventions, Teubert and Pinquart's (2011) review of 65 trials (where anxiety symptoms or diagnostic status was a primary or secondary outcome) found that, at post-test, targeted programs ( $k=36$ studies) were associated with a significantly higher mean ES (0.32) than universal programs (0.12), although this difference was no longer significant at follow-up (0.15 for universal and 0.23 for indicated) (0.23) [17]. In comparison, Werner-Seidler et al.'s (2017) review of 81 original randomised-controlled trials (RCTs), which also merged indicated and selective programs, found no significant differences in ES between these and universal programs for anxiety symptoms across all ages in the short, medium, or long terms [20]. Some targeted approaches performed no better than universal approaches in the short term or long term. In sum, reviews have produced mostly small post-test ES for targeted approaches. However, the merging of selective and indicated trials in these reviews is problematic as the former targets asymptomatic young people and the latter targets those with detectable elevated symptoms. Indicated interventions have the potential to be more effective than selective interventions given the scope for change in symptoms, and the targeting of especially responsive groups who are motivated to engage in the intervention [10]. To date, only one review has isolated the effect of school indicated interventions for reducing symptoms of anxiety. Neil and Christensen's (2009) review of 27 trials (published between 1987 and 2008) spanned universal $(k=16)$, indicated $(k=8)$, and selective $(k=3)$ programs for 5-19 year olds [16]. At post-test, $69 \%$ of the universal programs $(k=11)$ reported significant differences between the intervention and control conditions at post-test, with moderate-to-very large ES $(0.31-1.37)$. This compared to only $50 \%$ of indicated trials $(k=4)$ with small-to-moderate ES $(0.20-0.76)$. At followup, however, indicated trials outperformed universal programs. Out of six indicated trials that reported follow-up data (spanning 1-30 months), all but one (83\%) reported significant small-to-large effects (0.19-1.03). Out of the six trials of universal programs with follow-up data, 3 (50\%) reported significant small-to-moderate effects $(0.22-0.70)$. Thus, compared to universal programs, a higher proportion of indicated programs secured long-term symptom reduction, and some with larger effects (although the difference was not formally tested). However, the number of indicated trials was low and the quality poor.

A number of new trials of school indicated approaches for reducing anxiety symptoms have been conducted since Neil and Christensen [16]. Producing an updated ES for indicated school programs will progress the evidence for effective early intervention strategies for symptom reduction that can be delivered at a population level. The aim of our review was to (1) synthesise evidence on school indicated programs for reducing elevated (sub-clinical) anxiety symptoms in children and adolescents; (2) conduct a metaanalysis to identify their overall effects; (3) establish the duration of any effects; and (4) determine the relative effect 
of interventions according to control type, delivery agent, and intervention intensity.

\section{Methods}

\section{Data sources and searches}

We searched MEDLINE, EMBASE, PsycINFO, and the Cochrane Central Register of Controlled Trials, ClinicalTrials.gov, and PROSPERO from database inception to December 2019. Details of search strategies are provided in Suppl. Table 1. Grey literature was searched using Google Scholar. Reference lists of relevant studies and reviews were searched. Study authors were contacted in the case of missing or ambiguous information. The review protocol was developed using the Preferred Reporting Items for Systematic Reviews and Meta-analyses (PRISMA) statement guidelines [23] and was registered with PROSPERO [CRD42018087628 https://www.crd.york.ac.uk/prospero/ display_record.php?RecordID $=87628]$.

\section{Study selection}

Studies were eligible for this review if they were published in English and met the following PICOS inclusion criteria: $(P)$ Population children/adolescents aged 5-18 years with elevated levels of anxiety. Studies with both sub-clinical and (likely or confirmed) clinical populations were included only where the intervention aim was described as preventative rather than treatment. This decision accommodated any small variability in screening or diagnostic outcomes within a study, and the fact that many studies reported only symptom thresholds for inclusion and not upper thresholds for exclusion.

(I) Intervention school-based indicated prevention or early intervention programs for anxiety disorders, where school-based means endorsed by schools and delivered on school premises either face-to-face or online. Multi-tiered interventions were included if they contained an indicated intervention. Although our published protocol stated that we would include interventions that targeted depression and anxiety, Werner-Siedler et al. [20] recently reviewed indicated programmes for depression; hence, we then restricted our review to interventions for anxiety. (C) Comparator passive comparators (waitlist and no intervention) and active comparators (programs to control for non-specific aspects of anxiety treatment). (O) Outcome anxiety symptoms measured via validated self-rating or clinician-rated scales and/ or diagnostic outcomes (i.e., no longer meeting diagnostic thresholds showing symptom reduction). (S) Study design randomised-controlled trials.

\section{Outcome measures}

Our main outcome was reduction of anxiety symptoms and prevention of anxiety disorder progression, i.e., a change in symptoms that could represent prevention of disorder. Our primary outcome for efficacy was the mean change scores on anxiety symptoms in comparison to controls, based on self-rating or clinician-rated scales. Table 1 shows the primary outcome measures utilised in each study and included the Revised Children's Anxiety and Depression scale [24], the Spence Children's Anxiety Scale [25], the Revised Children's Manifest Anxiety Scale [26], the Screen for Child Anxiety Related Disorders [27], the State-Trait Anxiety Inventory [28], the Multidimensional Anxiety Scale for Children [29], the Child Behaviour Checklist [30], and the Social Phobia subscale [31]. Where a trial included more than one scale for anxiety symptoms, we used the scale with the greatest frequency across studies. Change in anxiety symptoms was also assessed in some studies via diagnostic interviews.

\section{Screening, data extraction, and quality assessment}

Three authors (SB and PM) and a medical student (independent of study) separately screened the titles and abstracts of studies against the inclusion criteria in the first round of searching (up to September 2018). In the updated search (up to December 2019), abstracts were separately screened by two authors (PM, ET). Independent full-text reviews were completed by SB and PM (first search) and ET, PM and SHJ (updated search). Studies were included if they met all of the inclusion criteria. Disagreements between reviewers were resolved through discussion. For the included studies, data were extracted, using a Cochrane data abstraction form, by one investigator (SB) and were independently checked by another (PM). Data extracted included study details (author, publication year, and country), participant details (age range, gender, sample size, and school type), methodology (design, unit of allocation), intervention characteristics (type, content, target, provider, parental involvement, frequency, duration, and indicators of acceptability), control group (type), and all reported outcomes (definition, time points, person measuring, summary estimates, fidelity, acceptability, and compliance). For the waitlist control studies, follow-up data were only extracted if the waitlist control group remained waitlist at follow-up. Risk of bias was assessed with the Cochrane Handbook Risk of Bias Assessment Tool [32]. Risk of bias was assessed independently by one investigator (SB) and a medical student, neither of whom were blinded to the review aim. As recommended by Cochrane, risk of bias was reported separately for each of the seven criteria, namely random sequence generation, adequate concealment of this sequence, blinding of participants/personnel, blinding of 
Table 1 Characteristics of included trials $(k=20)$ by date of publication

\begin{tabular}{|c|c|c|c|c|c|c|c|c|}
\hline Trial citation & Program & $\begin{array}{l}N \\
\text { Age (years) }\end{array}$ & Control & Delivery & Content & $\begin{array}{l}\text { Primary anxiety } \\
\text { measure }\end{array}$ & $\begin{array}{l}\text { Post-test effect } \\
\text { size* }\end{array}$ & $\begin{array}{l}\text { Follow-up } \\
\text { effect size } \\
\text { (months) }\end{array}$ \\
\hline $\begin{array}{l}\text { Kiselica et al. } \\
\text { [41] }\end{array}$ & $\begin{array}{l}\text { Stress inocula- } \\
\text { tion }\end{array}$ & $\begin{array}{l}48 \\
14-15\end{array}$ & NI & MHP + school MHP & CBT & STAI A-TRAIT & $-0.74^{\mathrm{a}}$ & $-1.01(1)^{\mathrm{c}}$ \\
\hline Dadds et al. [36] & Coping koala & $\begin{array}{l}128 \\
7-14\end{array}$ & NI & $\mathrm{MHP}+\mathrm{Grad}$ & CBT & RCMAS & 0.01 & $-0.05(6)$ \\
\hline $\begin{array}{l}\text { Mifsud and } \\
\text { Rapee [51] }\end{array}$ & Cool kids & $\begin{array}{l}91 \\
8-11\end{array}$ & WL & MHP + school MHP & CBT & SCAS & -0.35 & $-0.57(4)^{\mathrm{c}}$ \\
\hline $\begin{array}{l}\text { Bernstein et al. } \\
{[34,54]}\end{array}$ & $\begin{array}{l}\text { FRIENDS } \\
\text { (child + parent) }\end{array}$ & $\begin{array}{l}61 \\
7-11\end{array}$ & $\begin{array}{l}\text { FRIENDS child } \\
\text { only + WL }\end{array}$ & MHP + Grad & CBT & MASC $^{b}$ & 0.22 & $--0.04(6)$ \\
\hline $\begin{array}{l}\text { Gillham et al. } \\
\text { [39] }\end{array}$ & $\begin{array}{l}\text { Penn resiliency } \\
\text { program }\end{array}$ & $\begin{array}{l}44 \\
11-13\end{array}$ & NI & $\begin{array}{l}\text { Grad/ } \\
\text { researchers }\end{array}$ & CBT & RCMAS & -0.07 & $\begin{array}{l}-0.62(6)^{\mathrm{c}} \\
-0.79(12)^{\mathrm{c}}\end{array}$ \\
\hline Siu [47] & FRIENDS & $\begin{array}{l}47 \\
7-10\end{array}$ & WL & MHP & CBT & SCARED & $-1.48^{\mathrm{a}}$ & NA \\
\hline Hunt et al. [38] ${ }^{\mathrm{a}}$ & FRIENDS & $\begin{array}{l}260 \\
11-13\end{array}$ & NI & $\begin{array}{l}\text { School } \\
\text { MHP+Teacher }\end{array}$ & CBT & SCAS & $\begin{array}{l}\text { No control } \\
\text { group data }\end{array}$ & $\begin{array}{l}0.17(24) \\
-0.01(48)\end{array}$ \\
\hline Siu [48] & Theraplay & $\begin{array}{l}46 \\
\text { Mean } 7.8\end{array}$ & WL & MHP & Play therapy & $\begin{array}{l}\text { Internalis- } \\
\text { ing scale of } \\
\text { CBCL }\end{array}$ & $-2.40^{\mathrm{a}}$ & NA \\
\hline $\begin{array}{l}\text { Manassis et al. } \\
\text { [37] }\end{array}$ & Feelings club & $\begin{array}{l}148 \\
8-12\end{array}$ & $\mathrm{AC}$ & MHP + Grad & CBT & MASC $^{\mathrm{b}}$ & -0.06 & $-0.06(12)$ \\
\hline $\begin{array}{l}\text { Liddle and Mac- } \\
\text { millan }[43]^{\mathrm{a}}\end{array}$ & FRIENDS & $\begin{array}{l}51 \\
8-14\end{array}$ & WL & MHP & CBT & SCAS & $\begin{array}{l}\text { No control } \\
\text { group data }\end{array}$ & NA \\
\hline $\begin{array}{l}\text { Cooley-Strick- } \\
\text { land et al. [35] }\end{array}$ & FRIENDS & $\begin{array}{l}93 \\
8-12\end{array}$ & WL & MHP + Grad & CBT & RCMAS & 0.21 & NA \\
\hline Miller et al. [52] & FRIENDS & $\begin{array}{l}191 \\
9-12\end{array}$ & $\mathrm{AC}$ & Teacher + MHP/Grad & CBT & MASC & 0.08 & NA \\
\hline Nobel et al. [45] & Feelings Club & $\begin{array}{l}78 \\
8-11\end{array}$ & $\mathrm{AC}$ & MHP & CBT & MASC & 0.03 & NA \\
\hline $\begin{array}{l}\text { McLoone and } \\
\text { Rapee [44] }\end{array}$ & Cool Kids & $\begin{array}{l}152 \\
7-12\end{array}$ & Home + WL & School MHP & CBT & SCAS & -0.43 & $-0.27(12)$ \\
\hline $\begin{array}{l}\text { Sportel et al. } \\
\text { [33]; de Hullu } \\
\text { et al. [55] }\end{array}$ & $\begin{array}{l}\text { Cognitive } \\
\text { Behavioural } \\
\text { Group }\end{array}$ & $\begin{array}{l}240 \\
12-16\end{array}$ & $\mathrm{CBT}+\mathrm{NI}$ & MHP & CBT & $\begin{array}{l}\text { SP subscale of } \\
\text { RCADS }^{b}\end{array}$ & 0.16 & $\begin{array}{l}-0.41(6)^{\mathrm{c}} \\
-0.17(12) \\
-0.21(24)\end{array}$ \\
\hline Yulei et al. [50] & Cool Kids & $\begin{array}{l}59 \\
14-17\end{array}$ & WL & $\mathrm{MHP}+\mathrm{Grad}$ & CBT & SCAS & -0.45 & NA \\
\hline $\begin{array}{l}\text { Hadwin et al. } \\
\text { [40] }\end{array}$ & Cogmed-WM & $\begin{array}{l}40 \\
11-14\end{array}$ & $\mathrm{AC}$ & Computerised & WM training & RCMAS & -0.46 & $0.00(3)$ \\
\hline Lam [42] & MBCT-C & $\begin{array}{l}20 \\
9-13\end{array}$ & WL & $\mathrm{MHP}+\mathrm{Grad}$ & Mindfulness & RCADS & 0.22 & NA \\
\hline $\begin{array}{l}\text { Scholten et al. } \\
\text { [46] }\end{array}$ & $\begin{array}{l}\text { Dojo video } \\
\text { game }\end{array}$ & $\begin{array}{l}138 \\
11-15\end{array}$ & $\mathrm{AC}$ & $\begin{array}{l}\text { Computer- } \\
\text { ised + researcher }\end{array}$ & $\begin{array}{l}\text { ERT }+ \text { HRV } \\
\text { biofeedback }\end{array}$ & SCAS & -0.11 & $0.03(3)$ \\
\hline $\begin{array}{l}\text { Van Starrenburg } \\
\text { et al. [49] }\end{array}$ & Coping Cat & $\begin{array}{l}141 \\
7-13\end{array}$ & WL & $\mathrm{MHP}+\mathrm{Grad}$ & CBT & SCAS & $-0.58^{\mathrm{a}}$ & $-0.64(3)^{\mathrm{c}}$ \\
\hline
\end{tabular}

Mode of delivery: MHP Mental health professional, Grad graduate students. Program content: $C B T$ Cognitive behavioural therapy, $M B C T$-C mindfulness-based cognitive therapy for children, $W M$ working memory, $E R T+H R V$ emotion regulation training and heart rate variability. Control: $W L$ wait list, NI no intervention, $A C$ attention control. Anxiety outcome measures: RCADS Revised Children's Anxiety and Depression scale, SCAS Spence Children's Anxiety Scale, RCMAS Revised Children's Manifest Anxiety Scale, SCARED The Screen for Child AnxietyRelated Disorders), STAI A-TRAIT State-Trait Anxiety Inventory, MAS Multidimensional Anxiety Scale for Children, $C B C L$ Child Behaviour Checklist, SP subscale Social Phobia subscale. Follow-up effect sizes: NA not available (typically as waitlist controls had started the intervention by follow-up, so no true control group data available)

*Negative effect sizes indicate reduction in symptoms

${ }^{a}$ Excluded from the meta-analysis

${ }^{\mathrm{b}}$ Also administered post-intervention diagnostic interviews

${ }^{\mathrm{c}}$ Significant differences in anxiety scores between intervention and control groups 
outcome assessment, reporting of incomplete outcome data, selective reporting of data, and protection against contamination. All domains were scored as (1) low risk of bias, (2) unclear, or (3) high risk of bias. Disagreement was resolved through discussion. Study quality was rated high risk study where three or more criteria showed high risk of bias, as low risk where five or more items rates showed low risk of bias, and as moderate risk in all remaining situations.

\section{Statistical analysis}

A meta-analysis using a random-effects model was conducted (using the RevMan software version 5.3) as considerable heterogeneity was expected across studies. The inverse variance method was used to weight studies. Continuous outcomes are presented as standardised ES. Standardised ES estimates, referring to the difference between the intervention and control group at post-intervention and followup, were calculated using Hedge's $g$ (with 95\% confidence intervals), which includes an adjustment for small-sample sizes. Negative ES indicate a decrease, and positive ES indicate an increase, in mean levels of anxiety symptoms between time points of comparison. The $I^{2}$ statistic was used to test for homogeneity of ES by indicating heterogeneity in percentages, with $25 \%, 50 \%$, and $75 \%$ representing low, moderate, and high heterogeneity, respectively. Sub-group analyses were conducted for delivery agent, control type, and intervention intensity. Sensitivity analyses were conducted using only studies with a low risk of bias. A funnel plot was used to assess for small-study effects and publication bias. Egger's tests were conducted where asymmetry was apparent on visual inspection. Duval and Tweedie's trimand-fill procedure was conducted using the Comprehensive Meta-Analysis software (version 3.0 Biostat Inc.) to give an adjusted estimate of unbiased ES.

\section{Results}

\section{Study selection and characteristics}

The PRISMA flow diagram (see Fig. 1) details the studyselection process. In total, 2813 studies were identified, of which 266 were duplicates and removed, leaving 2547 studies for title and abstract screening, following of which 2421 studies were excluded leaving 126 studies for fulltext screening. Of these, 103 were excluded. Reasons for exclusion were dissertations; duplicates; not an indicated intervention; wrong population; not anxiety; treatment not prevention; not RCT; not school-based; different intervention; and still recruiting. This left 23 studies for inclusion, of which 20 were original studies.
Characteristics of study samples, intervention, delivery agent, control type, and primary anxiety measure are shown in Table 1. The 20 reviewed studies included 2076 participants. All studies targeted generalised or unspecified anxiety except for one [33] which focused on social and test anxiety. Four studies (20\%) used diagnostic screening interviews that led to the inclusion of children who met both clinical and sub-clinical thresholds for anxiety disorder [33-36]. We included these as the interventions still had a preventive purpose for those not meeting clinical thresholds. The remaining 16 studies (80\%) used self, parent, or teacher report to screen for baseline elevated anxiety symptoms. Screening tools and cut-off scores used to determine inclusion varied across studies. Whilst most studies utilised standardized measures at outcome, six (30\%) also utilised diagnostic interviews [33-38].

Studies randomised participants at the individual $(k=13$; 65\%) [35, 39-50], group $(k=1 ; 5 \%)$ [37], and school levels $(k=6 ; 30 \%)[33,34,36,38,51,52]$. With one exception (which was computerised) [46], all programs were delivered to groups, and program sessions (ranging from 40 to $90 \mathrm{~min})$ were usually conducted weekly $(k=17 ; 85 \%)[34$, 36-45, 47-52], also twice weekly $(k=2 ; 10 \%)$ [33], [46] or biweekly $(k=1 ; 5 \%)$ [35]. Only two programs included booster sessions.[34, 38] One study delivered a booster package of intervention prompts to the families of the intervention group children post-intervention [36]. Secondary outcomes included academic achievement [35][40, 41] and two studies also reported on intervention acceptability.[42, 48] Of the 19 programs delivered face-to-face, only seven (37\%) were subjected to fidelity assessments, using either independent ratings of audio or video recordings of the sessions $(k=4 ; 21 \%)[37,38,42,52]$ or had supervised sessions $(k=3 ; 16 \%)$. [34, 36, 49] Of the ten studies $(50 \%)$ that reported on compliance [33-36, 39, 40, 42, 44, 47, 51], all reported high completion rates with at least more than $50 \%$ of participants attending more than $50 \%$ of the sessions. Drop out during the intervention was only clearly reported in 12 studies, and ranged from no dropout to a drop out of $35 \%$ [32] (see Suppl. Table 1) for further study details including screening measures, nature of control conditions, number of parent and child sessions, and other indicators of compliance (session attendance and measure non-completion).

The risk of bias was high, although most studies had an unclear risk of selection bias due to inadequate reporting of the randomisation procedure. Risk of contamination was high in 14 studies (70\%) (students randomised to groups within schools) and low in six studies (30\%) (cluster randomisation). Blinding of participants and personnel was difficult as most studies had a wait list or no intervention control group $(k=15 ; 75 \%)$. Blinding of outcome assessment was not feasible in 14 studies $(70 \%)$ as self and parent report outcome measures were used. Studies which included 


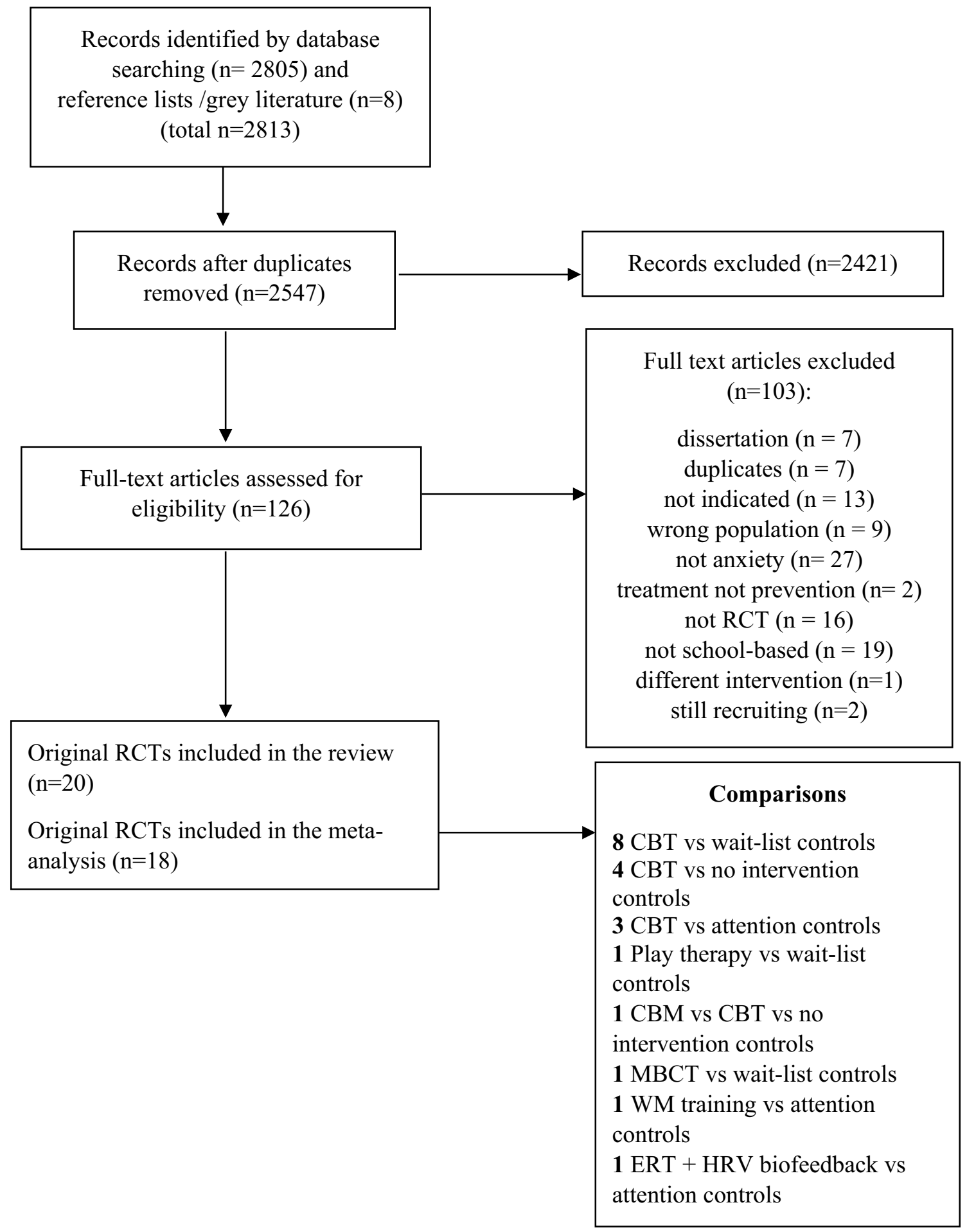

Fig. 1 Flow diagram of selection of studies for inclusion. $C B T$ cognitive behavioural therapy, $C B M$ cognitive bias modification, $M B C T$ - $C$ mindfulness-based cognitive therapy for children, $W M$ working memory, $E R T+H R V$ emotion regulation training and heart rate variability

an outcomes diagnostic interview generally had a low risk of detection bias as interviewers were blind to the participants' group $(k=4 ; 20 \%)$. For attrition bias, the risk was generally low. Nearly all studies had an unclear risk of reporting bias. The inter-rater reliability between the two independent reviewers for each risk of bias domain was adequate (Cohen's Kappa $>0.80$ for all risk of bias domains) (see Suppl. Fig. 1). 
A meta-analysis was conducted on the 18 studies with sufficient data to compare post-test differences in anxiety symptoms between intervention and control groups (Liddle and Macmillan [43] and Hunt et al. [38]were excluded due to inadequate data). Three studies had more than one comparison group. Therefore, for McLoone and Rapee [44], the school-based not home-based treatment group was included; for Bernstein, Layne, Egan, and Tennison [34], the childonly group was combined with the child + parent group to create a collapsed treatment group; and for Sportel, de Hullu, de Jong, and Nauta [33], we compared the CBT and no intervention control group, and for Hadwin et al. (2016) [40], we compared CBT (active control) and working memory training (intervention).

\section{Efficacy outcomes}

The overall post-test ES for change in anxiety symptoms was $g=-0.28(\mathrm{CI}=-0.50,-0.05)$ with large heterogeneity $\left(I^{2}=78 \%\right)(k=18)$. The ES for change in anxiety symptoms in the short term (0-6 months) was $g=-0.35(\mathrm{CI}=-0.58$, $-0.13)(k=9)$, in the medium term $(6-12$ months) was $\mathrm{g}=-0.24(\mathrm{CI}=-0.48,0.00)(k=4)$ and in the long term (>12 months) was $g=-0.01, \mathrm{CI}=-0.38,0.36)(k=2)$. Significant post-test differences between intervention and control groups were reported in 4 out of 18 studies (22\%) [41, 47-49] with medium-to-very large ES (based on Cohen [53]). At follow-up, significant medium-to-very large effects [10] were reported in 5 out of 11 studies with suitable data (46\%).[33, 39, 41, 49, 51] Five of the eighteen trials (28\%) reported small non-significant effects at either post-test or follow-up [34, 40, 44, 50][55]. Four trials (22\%) failed to find any effect at either post-test or follow-up [34, 43, 44, 54]. Findings from the four studies [35, 42, 45, 52] (and their follow-up publications) that reported post-test diagnostic interviews at 12 and $24 \mathrm{~m}$ showed that the odds of having an anxiety disorder were significantly lower in the intervention compared to the control group at $24 \mathrm{~m}(k=2, \mathrm{OR}=0.40$, $95 \% \mathrm{CI}=0.19,0.83)$ but not at $12 \mathrm{~m}(k=2, \mathrm{OR}=0.64,95 \%$ $\mathrm{CI}=0.34,1.18)$.

\section{Sub-group analyses}

We could not perform a sub-group analysis on age (child vs adolescent) due to an insufficient number of studies that included children only $(k=4)$ and adolescents only $(k=3)$. A sub-group analysis could also not be performed on delivery agent (as very few interventions were delivered by teachers, $k=2$ ) nor on program type (most were CBT based). Figure 2 depicts a Forest plot of the ES for sub-group comparisons between intervention types and control conditions on post-test anxiety scores. The only pooled studies with significant, small effects were those comparing CBT to waitlist $(g=-38)$.

No significant differences were found between control group type on the size of the effect $\left(\mathrm{Chi}^{2}=4.85, d f=2\right.$, $P=0.09$, wait list: $k=9, g=-0.53,95 \% \mathrm{CI}=-0.97$, -0.09 , attention control: $k=5, g=-0.00,95 \% \mathrm{CI}=-0.18$, 0.17 , no intervention: $k=4, g=-0.10,95 \% \mathrm{CI}=-0.44$, 0.24). Finally, no significant differences were found based on intervention intensity (delivered weekly, biweekly or twice weekly) on post-test effect size $\left(\mathrm{Chi}^{2}=4.58, d f=2\right.$, $P=0.10$; weekly: $k=15, g=-0.32,95 \% \mathrm{CI}=-0.59$, - 0.06; biweekly: $k=1, g=0.21,95 \% \mathrm{CI}=-0.20,0.62$; twice weekly: $k=2, g=-0.18,95 \% \mathrm{CI}=-0.48,0.13)$.

\section{Sensitivity analyses}

Sensitivity analyses were conducted on studies with a low risk of selection bias, a low risk of attrition bias, and a low risk of contamination (biases deemed most relevant to studies of this nature). Studies with a low risk of contamination had an ES of $g=0.03(k=5,95 \% \mathrm{CI}=-0.14,0.21)$. The ES for studies with a low risk of selection was $g=-0.21(k=4$, $95 \% \mathrm{CI}=-0.55,0.13)$ and for studies with low attrition bias was $g=-0.35(k=11,61 \% 95 \% \mathrm{CI}=-0.65,-0.05)$. Funnel plot asymmetry (see Suppl. Fig. 2) suggested a small publication bias [Egger's test: intercept: -4.10 [95\% CI - 7.59 to 0.61 ], $t=2.49, P=0.02$. Results were improved when the Duval and Tweedie's trim-and-fill method was used to adjust for funnel plot asymmetry ( $g$ was reduced from -0.28 to $-0.08)$. However, this method has been shown to perform poorly where high heterogeneity exists between studies.

\section{Discussion}

Early intervention to reduce symptoms of anxiety is an increasing global priority [56]. To our knowledge, ours is the first review to produce an ES for indicated schoolbased approaches for child and adolescent anxiety. We have addressed the limitations of previous meta-analyses which focused only on depression [12,21, 22], mixed settings [12, 15], and/or which merged selective and indicated interventions $[15,17,20]$. We found an overall small post-test ES for anxiety symptom reduction of -0.28 for intervention groups compared to controls. Large-to-very large effects at post-test were found in $22 \%$ of studies $(k=4)(-0.58$ to -2.40$)$ and at follow-up in $46 \%$ of studies $(k=5)(-0.41$ to -1.01$)$. Our findings are in line with Neil and Christensen [16]; although their data prohibited a formal meta-analysis, 50\% $(k=4)$ of their reviewed trials produced significant smallto-moderate post-test effects on anxiety symptoms ranging from $0.20-0.76$. That five (28\%) of our reviewed studies had a small but non-significant effect at either post-test or 


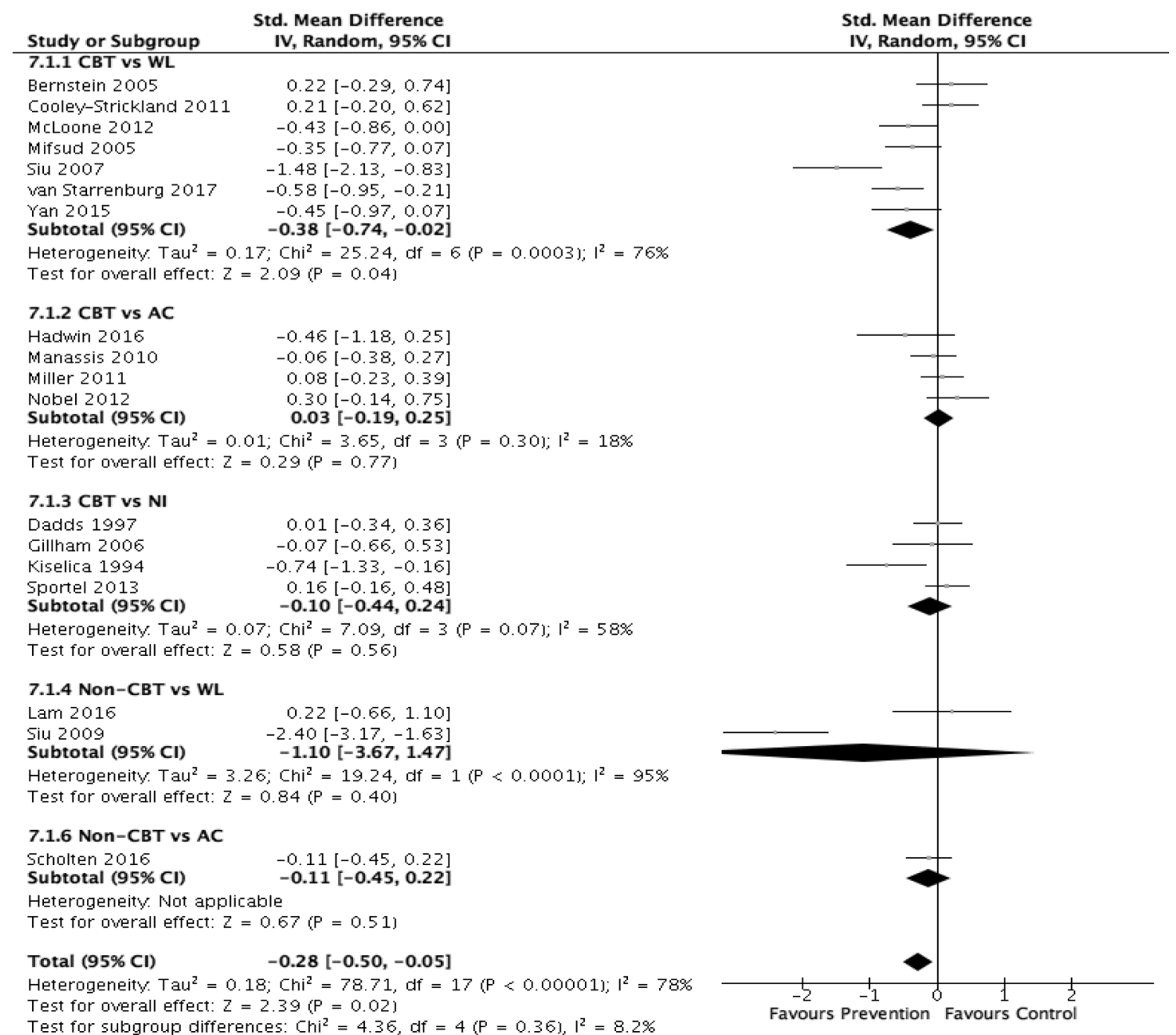

Fig. 2 Forest plot of effect sizes for comparisons between intervention (CBT or non CBT) and control conditions (waitlist, attention control, or no intervention) on post-intervention anxiety symptoms. The vertical line indicates the line of no effect, the horizontal lines

follow-up suggests that lack of power may be masking further potential positive effects.

Our post-test ES on anxiety symptoms is slightly smaller than those reported for universal programs [11-20]. However, concurring with Neil and Christensen [16], our review suggests that indicated programs may be more effective than universal programs over time. By 6 months, and based on nine studies, the post-test ES had increased from -0.28 to -0.35 , suggesting that young people may take time to benefit from programs. Four studies showed maintenance of a beneficial effect at 6-12 months $(-0.24)$, but this was no longer evident by $>12$ months. This may be because only two studies had sufficient data beyond 12 months, and further positive outcomes may have been missed as the studies with larger, significant post-test ES did not collect long-term outcomes [47, 48]. Good long-term outcomes were also evident in five studies in our review that utilised follow-up indicates the $95 \%$ confidence intervals, and the green dots represent the effect estimates from individual studies. Black diamonds indicate the pooled results of the studies

diagnostic interviews, and which found that the odds of having an anxiety disorder at 12 and 24 months was lower in the intervention group compared to controls. Whilst this was non-significant at 12 months, the odds became significant at 24 months, suggesting that some indicated prevention programs can prevent the onset of disorder. Thus, whilst there were only a small number of studies collecting longterm data from indicated programs, our review supports Neil and Christensen's [16] finding that indicated programs can produce good medium-to-long-term outcomes for anxiety reduction in children and adolescents.

Our reviewed studies produced a range of ES at post-test and follow-up. Several factors may be driving this variability, including content type, fidelity of implementation, inclusion of parents and booster sessions, participant engagement, sensitivity of outcome measures and trial quality. We did not find an effect of intervention intensity, although all bar 
three of our included studies were delivered weekly. Similar to Neil and Christensen [16], we did not find an effect of control group. The absence of an effect of control group suggests that the attention control conditions either did not impact anxiety outcomes or that there was insufficient evidence to detect a difference. Better attention controls, with high therapeutic potential [57], could help to determine whether general vs. specific factors have a role in anxiety prevention.

We could not conduct a sub-group analysis for age, delivery agent, or program type as there were too few studies. Notably, very few interventions were designed to be delivered by teachers. This may be because of the challenges in training teachers in mental health, compared to mental health professionals, for time-limited research studies and/or because of the lack of human and other resources available in schools for delivery by school staff. Findings on delivery agents in general are mixed, from no effect in universal programs [12], to better outcomes from teacher delivered indicated programs for anxiety [16] to better outcomes from mental health professionals for depression programs [20, 21, 58]. Age of participants, complexity of program, delivery support and level of teacher experience and understanding of anxiety vs depression may explain these outcomes.

This meta-analysis should be reviewed in the context of several limitations, including high heterogeneity in study design and methodology and poor study quality. Risk of contamination and publication bias were high. These limitations are common in trials of all program types $[19,20]$ and high contamination risk suggests that effects may actually be underestimated. Additionally, most studies relied on self-report measures. Although self-report measures do not have sufficient sensitivity and specificity to detect anxiety disorder [59,60], studies used validated self-report measures that can capture current interfering symptoms, and reduction of scores is meaningful [61]. Some studies in our review involved children with clinical levels of anxiety; we included these studies as the main study population was sub-clinical and the approach was prevention rather than treatment. Most studies reported symptom score cutoffs for inclusion but not upper symptom score cut-offs for exclusion, nor ranges of symptoms scores in the sample. Whilst sample means show that studies largely targeted sub-clinical young people, a minority of young people in the reviewed studies met, or would be likely to have met, clinical thresholds for disorder. Indicated programs may produce even larger effects if only those with high, sub-clinical levels of anxiety (rather than mild-moderate) were recruited. Thus, it is possible that some of the interventions were treatment rather than indicated prevention. However, in the absence of costly pre-screening for program entry, real-world school interventions are likely to attract young people with diverse symptoms. Thus, the reported ES are likely to be ecologically valid.

Future studies should explore the effects of different program intensities and the optimal age for intervention, considering the most critical and plastic periods of development. Although most programs were delivered by MHPs, this is unlikely to be a scalable and sustainable solution given costs and clinician availability. Teacher burden is already high. Evidence from low-and middle-income countries suggests that well-trained lay workers can be effective delivery agents of school mental health programs and future studies in high-income countries should examine their acceptability and effectiveness [62]. Robust assessments of fidelity remain infrequent and should be addressed. Whilst digitised delivery of interventions is growing and could protect fidelity, we need a better understanding of how young people engage digitally, independently, and remotely with mental health content. It is also unclear if and how parental involvement can enhance school-based interventions. Cluster randomisation, attention controls, and detailed descriptions of the allocation sequence generation and concealment would help to improve the quality of future trials of school indicated programs. Future studies should assess outcomes beyond 12 months. As children's ability to accurately selfreport anxiety symptoms may vary considerably across ages, studies should also use multi-informant assessment methods [60]. Adolescents may have different rates of diagnostic accuracy to younger children, but this has yet to be established. Greater use of diagnostic assessments would strengthen the emerging evidence that indicated approaches can prevent anxiety disorder onset. Pragmatic trials in schools are needed to build evidence on implementation, sustainability, effectiveness, and cost-effectiveness.

\section{Conclusions}

Recommendations for implementing school-based interventions should be based on the analysis of multiple factors including intervention cost, the personal and societal burden borne by individuals without intervention, and the likely ES of an intervention [10]. This review of schoolbased indicated programs for reducing symptoms of anxiety in children and adolescents has produced a favourable post-test ES that exceeds the estimates produced by others for universal prevention programs in schools and in other settings for youth internalising problems [11]. Even stronger effects were found at 6-12 months, which exceeded many ES produced from reviews of universal approaches in school for anxiety symptom reduction. However, the small number of studies with long-term follow-up data, and the considerable 
heterogeneity and high risk of bias in the reviewed trials means that the findings should be interpreted with caution.

Acknowledgements We wish to thank Dr Sayeed Haque for his statistical advice, Anita Phul for support with the search strategy, Anna Cunningham for assistance in retrieving papers, Student $J$ for screening studies and quality appraisal, and Aidan Mooney for additional checks on aspects of data extraction.

Author contributions SB and PM conceived the study and conducted the review. SHJ contributed to data checking. SB drafted an early version of the manuscript, and SHJ developed and wrote the manuscript for publication. EL assisted with updating the review. All authors have contributed equally to this paper, and have read and approved the final version of the manuscript.

\section{Compliance with ethical standards}

Conflict of interest The authors declare that they have no conflict of interest.

Ethical approval No ethical approval required.

Open Access This article is licensed under a Creative Commons Attribution 4.0 International License, which permits use, sharing, adaptation, distribution and reproduction in any medium or format, as long as you give appropriate credit to the original author(s) and the source, provide a link to the Creative Commons licence, and indicate if changes were made. The images or other third party material in this article are included in the article's Creative Commons licence, unless indicated otherwise in a credit line to the material. If material is not included in the article's Creative Commons licence and your intended use is not permitted by statutory regulation or exceeds the permitted use, you will need to obtain permission directly from the copyright holder. To view a copy of this licence, visit http://creativecommons.org/licenses/by/4.0/.

\section{References}

1. Polanczyk GV, Salum GA, Sugaya LS, Caye A, Rohde LA (2015) Annual research review: a meta-analysis of the worldwide prevalence of mental disorders in children and adolescents. J Child Psychol Psychiatry 56:345-365. https://doi.org/10.1111/jcpp.12381

2. Alonso J, Liu Z, Evans-Lacko S et al (2018) Treatment gap for anxiety disorders is global: Results of the World Mental Health Surveys in 21 countries. Depress Anxiety 35:195-208. https://doi. org/10.1002/da.22711

3. Ford T, Goodman R, Meltzer H (2003) Service use over 18 months among a nationally representative sample of British children with psychiatric disorder. Clin Child Psychol Psychiatry 8:37-51. https://doi.org/10.1177/1359104503008001006

4. James AC, James G, Cowdrey FA, Soler A, Choke A (2013) Cognitive behavioural therapy for anxiety disorders in children and adolescents. Cochrane Database Syst Rev 6:CD004690. https://doi.org/10.1002/14651858.CD004690.pub3

5. Andrews G, Issakidis C, Sanderson K, Corry J, Lapsley H (2004) Utilising survey data to inform public policy: comparison of the cost-effectiveness of treatment of ten mental disorders. Br J Psychiatry 184:526-533. https://doi.org/10.1192/ bjp.184.6.526
6. Feldner MT, Zvolensky M (2004) Prevention of anxiety psychopathology: a critical review of the empirical literature. Clin Psychol Sci Pract 11:405-424. https://doi.org/10.1093/clipsy.bph098

7. Rapee R (2008) Prevention of mental disorders: promises, limitations, and barriers. Cognit Behav Pract 15:47-52. https://doi. org/10.1016/j.cbpra.2007.08.001

8. Barrett PM, Pahl KM (2006) School-based intervention: examining a universal approach to anxiety management. J Psychol Couns Schools 6:55-75. https://doi.org/10.1375/ajgc.16.1.55

9. Haggerty RJ, Mrazek PJ (1994) Reducing risks for mental disorders: frontiers for preventive intervention research. National Academies Press, Washington

10. Dodge KA (2019) Annual research review: universal and targeted strategies for assigning interventions to achieve population impact. J Child Psychol Psychiatry. https://doi.org/10.1111/ jcpp. 13141

11. Tanner-Smith EE, Durlak JA, Marx RA (2018) Empirically based mean effect size distributions for universal prevention programs targeting school-aged youth: a review of meta-analyses. Prev Sci 19:1091-1101. https://doi.org/10.1007/s11121-018-0942-1

12. Ahlen J, Lenhard F, Ghaderi A (2015) Universal prevention for anxiety and depressive symptoms in children: a meta-analysis of randomized and cluster-randomized trials. J Prim Prev 36:387403. https://doi.org/10.1007/s10935-015-0405-4

13. Corrieri S, Heider D, Conrad I, Blume A, König HH, Riedel-Heller SG (2013) School-based prevention programs for depression and anxiety in adolescence: a systematic review. Health Promot Int 29:427-441. https://doi.org/10.1093/heapro/dat001

14. Fisak BJ, Richard D, Mann A (2011) The prevention of child and adolescent anxiety: a meta-analytic review. Prev Sci 12:255-268. https://doi.org/10.1007/s11121-011-0210-0

15. Lawrence PJ, Rooke SM, Creswell C (2017) Prevention of anxiety among at-risk children and adolescents - a systematic review and meta-analysis. Child Adolesc Mental Health 22:118-130. https:// doi.org/10.1111/camh.12226

16. Neil AL, Christensen H (2009) Efficacy and effectiveness of school-based prevention and early intervention programs for anxiety. Clin Psychol Rev 29:208-215. https://doi.org/10.1016/j. cpr.2009.01.002

17. Teubert D, Pinquart M (2011) A meta-analytic review on the prevention of symptoms of anxiety in children and adolescents. J Anxiety Disord 25:1046-1059. https://doi.org/10.1016/j.janxd is.2011.07.001

18. Stockings EA, Degenhardt L, Dobbins T et al (2016) Preventing depression and anxiety in young people: a review of the joint efficacy of universal, selective and indicated prevention. Psychol Med 46:11-26. https://doi.org/10.1017/S0033291715001725

19. Waldron SM, Stallard P, Grist R, Hamilton-Giachritsis C (2018) The 'Long-Term' effects of universal school-based anxiety prevention trials: a systematic review. Mental Health Prev 11:8-15. https://doi.org/10.1016/j.mhp.2018.04.003

20. Werner-Seidler A, Perry Y, Calear AL, Newby JM, Christensen H (2017) School-based depression and anxiety prevention programs for young people: a systematic review and meta-analysis. Clin Psychol Rev 51:30-47. https://doi.org/10.1016/j.cpr.2016.10.005

21. Merry SN, Hetrick SE, Cox GR, Brudevold-Iversen T, Bir JJ, McDowell H (2012) Psychological and educational interventions for preventing depression in children and adolescents. Cochrane Database of Syst Rev 12:CD003380. https://doi. org/10.1002/ebch.1867

22. Calear AL, Christensen H (2010) Systematic review of schoolbased prevention and early intervention programs for depression. J Adolesc 33:429-438. https://doi.org/10.1016/j.adole scence.2009.07.004

23. Moher D, Shamseer L, Clarke M et al (2015) (2011) Preferred reporting items for systematic review and meta-analysis 
protocols (PRISMA-P) 2015 statement. Syst Rev 4:1. https:// doi.org/10.1186/2046-4053-4-1

24. Chorpita BF, Ebesutani C, Spence SH (2015) Revised Children's Anxiety and Depression Scale. Hämtad fran. https:// www.corc.uk.net/outcome-experience-measures/revised-child rens-anxiety-and-depression-scale-and-subscales/. Accessed 1 June 2020

25. Spence SH (1997) Structure of anxiety symptoms amongst children: a confirmatory factor-analytic study. J Abnorm Psychol 106:280-297. https://doi.org/10.1037/0021-843X.106.2.280

26. Lowe PA (2015) The revised Children's manifest anxiety scale-second edition short form: examination of the psychometric properties of a brief measure of general anxiety in a sample of children and adolescents. J Psychoeduc Assess 33:719-730. https://doi.org/10.1177/0734282915580763

27. Muris P, Merckelbach H, Van Brakel A, Mayer AB (1999) The revised version of the screen for child anxiety related emotional disorders (SCARED-R): further evidence for its reliability and validity. Anxiety Stress Coping 12:411-425. https://doi. org/10.1080/10615809908249319

28. Spielberger, C.D. (2010). State-Trait Anxiety Inventory. In: Weiner IB, Craighead WE (eds) The corsini encyclopedia of psychology. https://doi.org/10.1002/9780470479216.corps y0943

29. March JS, Parker JD, Sullivan K, Conners SP (1997) The Multidimensional Anxiety Scale for Children (MASC): factor structure, reliability, and validity. J Am Acad Child Adolesc Psychiatry 36:554-565. https://doi.org/10.1097/00004583-19970 4000-00019

30. Achenbach TM (1991) Integrative guide for the $1991 \mathrm{CBCL} / 4-18$, YSR and TRF profiles. University of Vermont, Burlington

31. Turner SM, Beidel DC, Dancu CV, Stanley MA (1989) An empirically derived inventory to measure social fears and anxiety: the social phobia and anxiety inventory. Psychol Assess 1:35-40. https://doi.org/10.1037/1040-3590.1.1.35

32. Higgins JPT, Altman DG, Gøtzsche P et al (2011) The Cochrane Collaboration's tool for assessing risk of bias in randomised trials. BMJ 343:d5928. https://doi.org/10.1136/bmj.d5928

33. Sportel BE, Hullu E, de Jong PJ, Nauta MH (2013) Cognitive bias modification versus CBT in reducing adolescent social anxiety: a randomised controlled trial. PLoS ONE 8:e64355. https://doi. org/10.1371/journal.pone.0064355

34. Bernstein GA, Layne AE, Egan EA, Tennison DM (2005) Schoolbased interventions for anxious children. J Am Acad Child Adolesc Psychiatry 44:1118-1127. https://doi.org/10.1097/01. chi.0000177323.40005.a1

35. Cooley-Strickland MR, Griffin RS, Darney D, Otte K, Ko J (2011) Urban African American youth exposed to community violence: a school-based anxiety preventive intervention efficacy study. J Prev Interv Community 39:149-166

36. Dadds MR, Spence SH, Holland DE, Barrett PM, Laurens KR (1997) Prevention and early intervention for anxiety disorders: a controlled trial. J Consult Clin Psychol 65:627-635. https://doi. org/10.1037/0022-006X.65.4.627

37. Manassis K, Wilansky-Traynor P, Farzan N, Kleiman V, Parker K, Sanford M (2010) The feelings club: randomized controlled evaluation of school-based CBT for anxious or depressive symptoms. Depress Anxiety 27:945-952. https://doi.org/10.1002/da.20724

38. Hunt C, Andrews G, Sakashita C, Crino R, Erskine A (2009) Randomized controlled trial of an early intervention programme for adolescent anxiety disorders. Aust N Z J Psychiatry 43:300-304. https://doi.org/10.1080/00048670902721152

39. Gillham JE, Reivich KJ, Freres DR et al (2006) School-based prevention of depression and anxiety symptoms in early adolescence: a pilot of a parent intervention component. School Psychol Q 21:323. https://doi.org/10.1521/scpq.2006.21.3.323
40. Hadwin JA, Richards HJ (2016) Working memory training and CBT reduces anxiety symptoms and attentional biases to threat: a preliminary study. Front Psychol. https://doi.org/10.3389/fpsyg 2016.00047

41. Kiselica MS, Baker SB, Thomas RN, Reedy S (1994) Effects of stress inoculation training on anxiety, stress, and academic performance among adolescents. J Couns Psychol 41:335

42. Lam K (2016) School-based cognitive mindfulness intervention for internalizing problems: pilot study with Hong Kong elementary students. J Child Fam Stud 25:3293-3308. https:// doi.org/10.1007/s10826-016-0483-9

43. Liddle I, Macmillan S (2010) Evaluating the FRIENDS programme in a Scottish setting. Educ Psychol Pract 26:53-67. https ://doi.org/10.1080/02667360903522785

44. McLoone JK, Rapee RM (2012) Comparison of an anxiety management program for children implemented at home and school: lessons learned. School Mental Health 14:231-242. https://doi. org/10.1007/s12310-012-9088-7

45. Nobel R, Manassis K, Wilansky-Traynor P (2012) The role of perfectionism in relation to an intervention to reduce anxious and depressive symptoms in children. J Ration Emotive Cognit Behav Ther 30:77-90. https://doi.org/10.1007/s10942-011-0133-5

46. Scholten H, Malmberg M, Lobel A, Engels RCME, Granic I (2016) A randomized controlled trial to test the effectiveness of an immersive 3D video game for anxiety prevention among adolescents. PLoS ONE 1:e0147763. https://doi.org/10.1371/journ al.pone. 0147763

47. Siu AFY (2007) Using FRIENDS to combat internalizing problems among primary school children in Hong Kong. J Cognit Behav Psychother 7:11-26

48. Siu AFY (2009) Theraplay in the Chinese world: an intervention program for Hong Kong children with internalizing problems. Int J Play Ther 18:1-12. https://doi.org/10.1037/a0013979

49. van Starrenburg MLA, Kuijpers RCMW, Kleinjan M, Hutschemaekers GJM, Engels RCME (2017) Effectiveness of a cognitive behavioral therapy-based indicated prevention program for children with elevated anxiety levels: a randomized controlled trial. Prev Sci 8:31-39. https://doi.org/10.1007/s11121-016-0725-5

50. Yulei Y, Shanshan W, Miao T, Jianping W, Qiuyuan X (2015) Effects of group cognitive-behavioral therapy on adolescent anxiety symptoms. Zhongguo Xinli Weisheng Zazhi 9:10-15

51. Mifsud C, Rapee RM (2005) Early intervention for childhood anxiety in a school setting: outcomes for an economically disadvantaged population. J Am Acad Child Adolesc Psychiatry 4:996-1004. https://doi.org/10.1097/01.chi.0000173294.13441

52. Miller LD, Laye-Gindhu A, Liu Y, March JS, Thordarson DS, Garland EJ (2011) Evaluation of a preventive intervention for child anxiety in two randomized attention-control school trials. Behav Res Ther 49:315-323. https://doi.org/10.1016/j.brat.2011.02.00

53. Cohen J (1988) Statistical power analysis for the behavioral sciences, 2nd edn. Lawrence Erlbaum, Hillsdale

54. Bernstein GA, Bernat DH, Victor AM, Layne AE (2008) Schoolbased interventions for anxious children: 3-, 6-, and 12-month follow-ups. J Am Acad Child Adol Psychiatry 47:1039-1047. https://doi.org/10.1097/CHI.ob013e31817eecco

55. de Hullu E, Sportel BE, Nauta MH, de Jong PJ (2017) Cognitive bias modification and CBT as early interventions for adolescent social and test anxiety: two-year follow-up of a randomized controlled trial. J Behav Ther Exp Psychiatry 55:81-89. https://doi. org/10.1016/j.jbtep.2016.11.011

56. World Health Organisation Mental Health Action Plan 20132020. https://www.who.int/mental_health/publications/actio n_plan/en/. Accessed 29 May 2019

57. Jensen PS, Weersing R, Hoagwood KE, Goldman E (2005) What is the evidence for evidence-based treatments? A hard look at 
our soft underbelly. Mental Health Serv Res 1:53-74. https://doi. org/10.1007/s11020-005-1965-3

58. Stallard P (2013) School-based interventions for depression and anxiety in children and adolescents. Evid Based Mental Health 16:60-61. https://doi.org/10.1136/eb-2013-101242

59. Reardon T, Spence SH, Hesse J, Shakir A, Creswell C (2018) Identifying children with anxiety disorders using brief versions of the Spence Children's Anxiety Scale for children, parents, and teachers. Psychol Assess 30:1342-1355. https://doi.org/10.1037/ pas0000570

60. Evans R, Thirwall K, Cooper P, Creswell C (2017) Using symptom and interference questionnaires to identify recovery among children with anxiety disorders. Psychol Assess 29:835-843. https ://doi.org/10.1037/pas0000375

61. Gillham AJ, Shatté K, Reivich JE (2001) Needed for prevention research: Long-term follow up and the evaluation of mediators, moderators, and lay providers. Prev Treat. https://doi. org/10.1037/1522-3736.4.1.49c

62. Fazel M, Patel V, Thomas S, Tol W (2014) Mental health interventions in schools in low-income and middle-income countries. Lancet Psychiatry 1:388-398. https://doi.org/10.1016/S2215 -0366(14)70357-8 